\title{
The Journal of the American Medical Association
}

VoL. XXXV.

CHICAGO, ILLINOIS, DECEMBER 29, 1900.

No. 26.

\section{Driginal Articles.}

\section{TREATMENT OF INJURIES TO THE URETERS.* BYRON B. DAVIS, M.D.}

Irofessor of Surgical Pathology and Clinical Surgery, Omaha Medical College; Surgeon to Immanuel Hospital, etc. OMAHA, NEB.

Feb. 22, 1900, Mrs. B. M., aged 38 years, housewife, with no children, reported that two months before, she began having morning nausea, and supposed she was pregnant, but menstruation continued. Thursday, February 15, one week before I saw her in consultation with Drs. Hoffman and Detwiler, what seemed to be a normal menstruation came to an end, and she felt perfectly well. 'The next morning she felt a sudden sharp pain in the lower abdomen, radiating toward the left side, and she had to lie down. A little more menstrual blood made its appearance, and she has had a slight flow ever since, but nothing has been found to suggest a miscarriage, and no decidual tissue has been discovered. Pain has been at times severe and cutting. Since February 18, Drs. Hoffman and Detwiler have seen her daily and her temperature has never been above normal, nor has her pulse suggested shock. A mass in the left lower quadrant of the abdomen has been increasing rapidly in size.

When seen by the writer, February 22 , she had a normal temperature and pulse, and was a spare but apparently wellnourished woman. A slightly nodular mass was found in the left inguinal region, extending an inch above the umbilicus and more than an inch to the right of the median line. The mass also presented behind the cervix, tense, but slightly fluctuating. It was not very painful on pressure.

Three explanations were suggested to account for the condition: 1, a pelvic abscess caused by an undiscovered abortion; 2 , a ruptured tubal pregnancy; 3 , an intraligamentous cyst, growing rapidly because of some change in its circulation brought about by trauma or pressure. The first supposition was untenable because of the absence of fever or increased pulse-rate. Neither was the pain significant of a rapid pus formation. Extrauterine pregnancy seemed to also be negatived from absence of severe collapse, from the fact that the mass was firmer than we usually find in such cases, and from the somewhat nodular form of the growth. The most plausible explanation was that we had to do with a rapidly-growing intraligamentous cyst.

Operation was performed Feb. 26, at 9 a. m., at Immanuel Hospital. Drs. Hotiman and Detwiler assisted, and Dr. Peterson, the house surgeon, gave ether. On opening the abdomen, an adherent, thick-walled cyst was found, the intestines and omentum being firmly adherent to it and to each other everywhere. After loosening the adhesions, some of which were so vascular they had to be ligated and cut, the left tube was found to be tensely stretched over the tumor, proving it to be intraligamentous. As it grew, it had burrowed up behind the peritoneum. The contents-clear serum-were removed by aspiration.

By hard work, lasting an hour, the cyst-wall was completely enucleated, but during this process several clamps had to be put on to stop the free bleeding. Sufficient care was supposed to

* Presented to the Section on Surgery and Anatomy. at the Fifty-first Annual Meeting of the American Medical Association, held at Atlantic Cit.y, N. J., June 5-8, 1900. have been taken to avoid the ureter, but, after clearing the mass away, a suspicious-looking vessel was found hanging loose in the pelvis. A small Kelly's probe was introduced into its lumen, and passed directly into the bladder. This portion was about $3 \frac{1}{2}$ inches long, so search was made for the upper fugment, and it was found within the grasp of a pair of forceps so near the median line that no thought had been present of being in a dangerous locality. The probe passed directly up to the pelvis of the kidney. I therefore united the severed ends of the ureter by an end-in-end anastomosis. The lower end was split for a distance of a centimeter, by introducing one blade of a pair of fine scissors into the lumen. Next, with a fine catgut suture, threaded with a needle at each end, the needles were passed side by side into the lumen of the upper fragment, and from within out through the wall of the ureter about one-eighth inch from the open end. These needles were about one-fifth the circumference of the duct apart, and at an equal distance from the free end. Next the needles were introduced into the lumen of the lower fragment and earried from within out through its wall at a distance of about one-half inch below the lower angle made by the slit in the wall; the needles here being the same distance apart as in the upper portion. (See Fig. 1.) With an assistant holding the free angles made by the slit in the lower fragment by means of small artery forceps, the opening was made more patulous and the duct steadied, while the upper fragment was drawn down within the lower. The catgut suture was then tied, and this held the upper fragment securely invaginated within the lower. With two additional catgut sutures the slit made in the lower fragment was repaired. (See Fig. 2.) The ureter was then covered as well as possible with peritoneum and the abdominal wall closed, but with a strip of gauze extending from the lower angle of the wound to the point of union of the duct.

The patient suffered from shock but quickly rallied. The first urine drawn from the bladder after the operation contained a slight amount of blood. At $6 \mathrm{p}$. m. of the day of operation, the temperature reached 99.4 degrees, the highest it rose at any time. March 3, the fifth day after the operation, the gauze drain was removed. No leakage of urine had occurred. By March 14 the wound was nicely healed and the patient was allowed to sit up. On March 22, to test the patulency of the ureter, a Harris "segregator" was used, and in thirty minutes 18 c.c. of urine was collected from the left-injured-kidney and 16 c.c. from the right. Phosphates were in excess, but otherwise the urine from each side was normal. On March 23, the patient was discharged from the hospital, and on May 29 was doing well, having gained several pounds in weight, and her urine being normal.

The possibility of injury to the ureter in intrapelvic operations has been recognized since the beginning of abdominal operations, but the full extent of this danger seems not to have been fully appreciated until the present decade. Could the whole truth be known, many cases of death reported as "shock" or "exhaustion" would have to be charged to "accidental injury to the ureter." Within a short time the writer learned of a woman dying from suppression of the urine shortly after an abdominal operation. Autopsy revealed the fact that both ureters had been tied and cut. Had no autopsy been secured, 
this would probably have been reported as a case of the "pernicious effects of the anesthetic." By making a regular practice of careful review after every complicated intrapelvic operation, one would not be likely to close the abdomen without discovering such an injury.

'The manner of dealing with a severed ureter, whether it be accidental or because of disease, will depend on the point of division. In the past the treatment advised and occasionally carried out was to remove the corresponding kidney, a measure so radical and so destructive that it had little except necessity to commend it. If the opposite kidney happened to be sound and the patient survived the shock of a major operation after having already endured a prolonged and difficult one, the result was satisfactory, although a person with only one kidney is greatly handicapped. To avoid so radical a procedure, when it was shown by the experiments of $R$. Harvey Reed and others that ligation of the ureter produced atrophy of the corresponding kidney, this was deemed a distinct gain, and was thought to mark an epoch in the treatment of this class of injuries. But it has been found that the results of ligation are not always the same, that atrophy does not always follow, and, if it does, the patient has the serious disability of having only one functionating kidney.
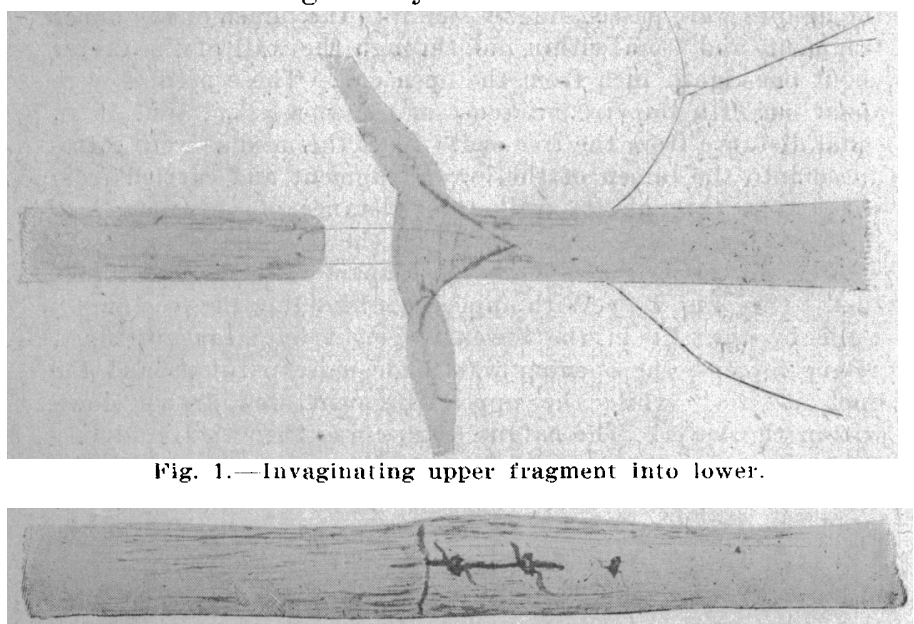

Fig. 2.-.-Anastomosis completed.

Stitching the severed ureteral end to the skin and thus establishing a permanent urinary fistula is less dangerous, so far as immediate results are concerned, but the disagreeable, not to say disgusting. condition in which the patient with such a fistula is left is enough to condemn it. In addition to the irritation of the skin and the foul odor which prevents the patient from mingling with his fellows, there is constant danger of an ascending infection to the pelvis of the kidney. This procedure has justly fallen into disuse and only the circumstance of this being the only procedure possible would warrant a surgeon in adopting it.

Much experimental work has been done to devise a safe and satisfactory method of implanting the severed ureteral end into the rectum or other part of the alimentary canal, but thus far it has not proved itself of value. Notwithstanding the apparent success of Martin's recent case of implantation of both ureters into the rectum with removal of the bladder for carcinoma, most surgeons would hesitate to follow his example. Theoretically, by imitating the oblique method by which the ureter enters the bladder, it was thought that infection of the kidney might be avoided, but the results in experiments on animals have not been encouraging. Dr. Reuben Peterson's general conclusions wele that the primary mortality of the uretero-intestinal anastomosis, both in experimental work on animals and in man, was exceedingly high; that all efforts to prevent ascending renal infection in animals or in man had proved futile. and that the operation was unjustifiable, either for the purpose of making the patient more comfortable or for malignant disease of the bladder. Van Hook ${ }^{1}$ says: "Implantation of the ureter into the intestines at any point is, in the writer's judgment, absolutely contraindicated, because of the certainty of infection of the ureter and its kidney." There is only one condition where implantation of the ureter into the intestine would seem justifiable until: 1 , the immediate mortality is reduced, and 2 , the dangers of an ascending infection to the pelvis of the kidney are lessened. The condition referred to is where the ureter is severed so high up that the proximal end can not be implanted into the bladder, and where there is so much loss of substance that the severed ureteral ends can not be united without too great tension. With this condition present, oblique implantation of the ureter into the colon would be preferable to the establishment of an external urinary fistula, to nephrectomy or to ligation of the ureter.

Implantation of the ureter into the vagina has little to commend it unless, by a plastic operation, it can be made to serve the purpose of a reservoir for the urine and its natural functions be abolished. Except in the event of total ablation of the bladder, no condition that warrants such an operation is conceivable. Even then one might follow the lead of Nichaus, who successfully implanted the ureter into the urethra!

But two practicable methods are left for disposing of an accidentally severed ureter: Implantation of the proximal end into the bladder and uretero-ureteral anastomosis. The former is only applicable in those cases in which the division occurs low down. In order to prevent retroflux of urine when the bladder contracts, and to minimize the dangers of an ascending infection, the method uniformly adopted is to imitate the natural manner in which the ureter enters the bladder. Novaro, Kelly, Penrose, Price, King and Baldy are some of the men who have successfully implanted the ureter into the bladder.

J. F. Baldwin ${ }^{2}$ reported the details of a case in which he excised $1 \frac{1}{2}$ inches of the right ureter while performing a hysterectomy. The lower end was too short for uretero-ureteral anastomosis. The proximal end, under considerable tension. was implanted into the bladder. To lessen the tension the bladder was drawn upward by suturing it to the broad ligament. His patient recovcred.

The first to perform the operation of uretero-ureteral anastomosis was Schopf, in 1886. The man who has probably done most to perfect a technique is Weller Van Hook, whose method of ligation of the distal end and implantation of the proximal end through a lateral longitudinal slit into the distal portion, has been practiced successfully in a number of eases. In summing up the advantages of his method, Van Hook says: 1. The urine is made to pass through its normal channel. 2. Healing takes place at once, without even temporary loss of function or a temporary fistula. 3. No stenosis occurs, even after a long interval of time. 4. The ureter can always be united, if accidentally injured at any operation. with materials always at hand. 5. Leakage can not occur, because the upper extremity of the ureter acts as an obdurator to the lower portion of the tube. 6 .

1. Internat. Text-Book of Surg., 11, p. 572.

2. Phila. Med. Jour., Nov. 29, 1898.

3. Internat. Text-Book of Surg., iv. p. 572. 
Scar-contraction can never injuriously diminish the Iumen of the tube, because the scar which encircles the ureter after union by this method is equal in length to twice the extent of the incision in the side of the lower ureteral stump.

'Transierse suture is difficult and seems likely to be followed by stricture, unless an effort is made, by splitting the ends, to increase the lumen. Hochenegg is reported to have made use of this method. Bovée reports having successfully employed direct suture after having divided the ends obliquely.

l'aggi is said to have been the first to propose end-inend anastomosis, but I have been unable to find a description of his method or the technique of the method as carried out by others. 'The method of end-in-end anastomosis as carried out in this case seems to have all the advantages of Van Hook's method, with one or two additional: 1. It is simple and more quickly done. 2. There is less waste of substance, and it would be available when the Van Hook method would produce too great tension, or where the distal fragment is so short that union by Van Hook's method could not be made. It is much quicker, simpler and surer than by transverse suture or oblique end-to-end suture as employed by Bovée.

When division of the ureter occurs, the following would seem to be the rational mode of choosing the method of repair: 1 . When possible to perform ureteroureteral anastomosis this is the preferable procedure: end-in-end anastomosis, as employed in this case, seeming to me simpler and better. 2. When the distal portion is too short for uretero-ureteral anastomosis, implantation into the bladder should be performed. 3. When there is too much loss of substance to permit ureteroureteral anastomosis, and the proximal end can not be brought down to the bladder even with the assistance of a diverticulum of the bladder, as devised by Van Hook. the procedure with the least objection is probably implantation into the colon.

\section{EPISPADTC EXSTROPHY OF THE BI ADDER COMPLETE.* \\ AP MORGAN VANCE. M.D. \\ LOUISVILLE, KY.}

Dr. Rudolph Matas, ${ }^{1}$ at the last meeting of the AssoCration, gave such a complete résumé of the subject that it would be simply repetition to now enter into a general discussion of the topic-and in his conclusions favored Maydl's operation, holding that the autoplastic operations could be little more than palliative, and never satisfactory. His paper decided me to report a case and exhibit the patient, showing the result of autoplastic work. I do not know how original the procedure to be described may be, though I have been unable to find a report of such a one.

Mr. J. McE., aged 17 years, was referred to me by Dr. J. M. Mathews on Oct. 26, 1897. He had come to Louisville to obtain a urinal and, if possible, get relief from the miserable condition of complete bladder exstrophy. Fig. 1 shows the condition before operation.

He was fairly well developed for his age, but his life was rendered unendurable as the result of excoriations from the urine and the contact of the clothing with the bulging posterior bladder wall, where ulceration with the deposit of calcareous material around the edges rendered the local condition horrible in the extreme. On Nov. 1,

* Presented to the Section on Surgery and Anatomy, at the Fifty-first Annual Meeting of the American Medical Association, held at Atlantic City, N. J., June 5-8, 1900

1. THE Journat, xxxili, p. 260.
1897, the first operation was done. This was much too soon, as five days was not sufficient to get the parts into proper condition, but it was done at this time because his family physician, Dr. Cowen, of Girard, Ill:, who (ame with him, wished to return.

The epispadic penis was very short and drawn back into a sulcus at the lower part of the opening. The posterior bladder wall, with discharging ureters, presented a convex surface far in front of the abdominal plane, about the size of the half of a baseball or largerwhen recumbent or in the Trendelenburg position a concave sulcus of corresponding dimensions presented.

The first step in the operation, as illustrated by Fig. 2, was to score as deeply as possible in a triangular manner.

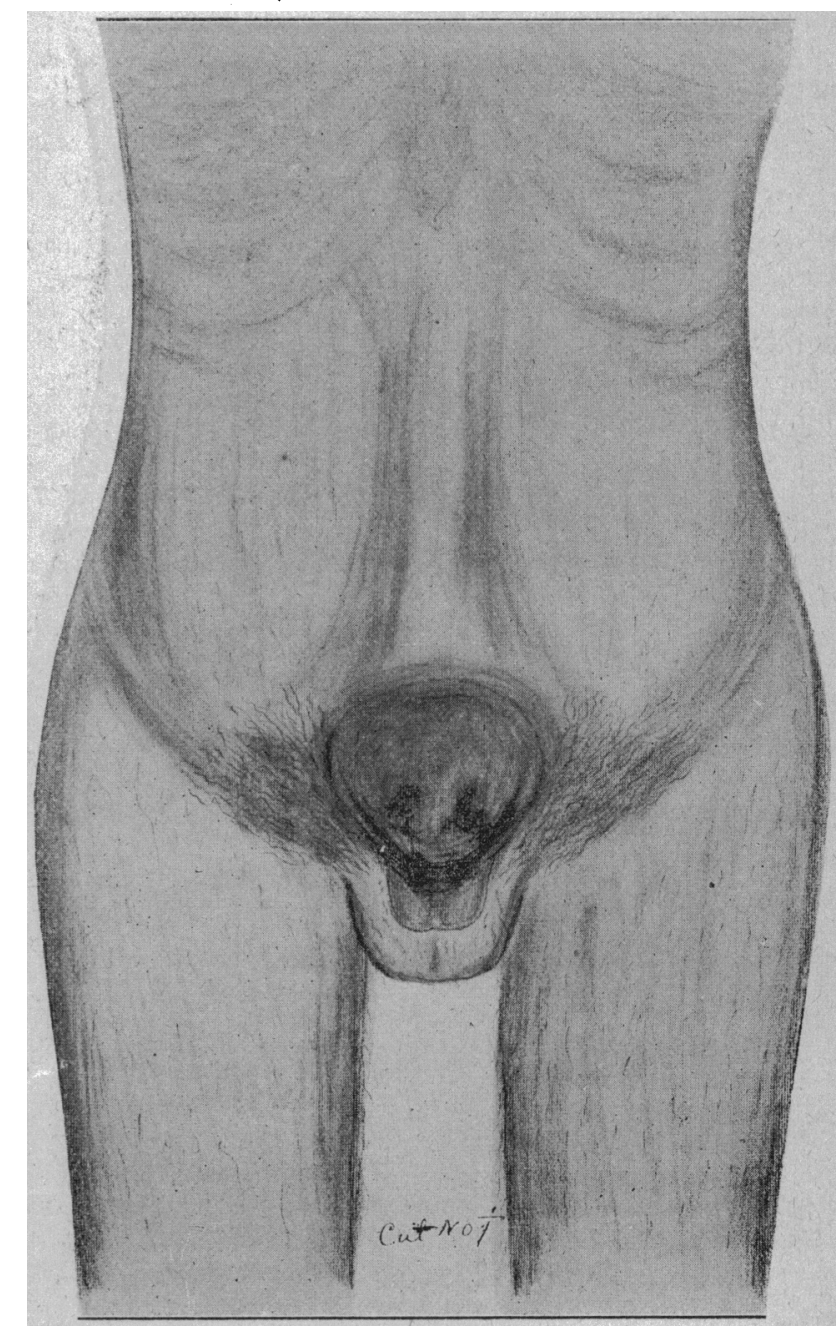

Figure: 1 .

wide out into the iliac regions, then with strong forceps to drag upward and inward, still further loosening the tissues thus freed. bringing the mucous membrane along with the skin. When this loosening and stretching had been done sufficiently to allow the separated pudendal structure to be brought together in the middle line, the edges down to the end of the penis were vivified by snlitting and brought together by two sets of sutures: one line of buried chromicized catgut, No. 1, which inverted the inner or mucous membrane side, and another of interrupted silkworm-gut which everted the outer or skin side. Over this a dressing of collodion and cotton was applied. The button-hole-like opening left above was used to drain the urine away, the patient being required to maintain the Trendelenburg position. No 